\title{
Stefan Nacke
}

\section{Kultur und Weiterbildung Culture and Education}

http://doi.org/10.1515/bd-2019-0030

Zusammenfassung: Der Beitrag plädiert für einen weiten Kulturbegriff. Die Bildungsexpansion der vergangenen Jahrzehnte hat zu einem neuen Klassenantagonismus geführt, indem es um gesellschaftliche Teilhabe durch kulturelle Teilhabe geht. Angesichts einer Kulturalisierung von Politik muss Kulturpolitik ihre gesellschaftspolitische Dimension wahrnehmen und neue bildungs- und weiterbildungspolitische Allianzen suchen.

Schlüsselwörter: Kulturbegriff, Kulturpolitik, Gesellschaftspolitik, Weiterbildung, Klassengesellschaft, Bildungsexpansion, Populismus

Abstract: The article argues for a broad concept of culture. The educational expansion in recent decades has created new class antagonisms by putting participating in society on a level with cultural participation. Given this 'culturalisation' of politics, it is important that cultural policies reinforce their sociopolitical dimension to build new alliances, particularly with regard to education and training policies.

Keywords: Concept of culture, cultural policies, social policies, continuing education, class society, expansion of education, populism

\section{Einleitung}

Kultur ist die Möglichkeit zur Identifikation mit Sinnzusammenhängen, mit Geschichte. Kultur ermöglicht die Beschreibung einer Identität. Gemeinschaften werden über gemeinsame Kultur integriert. Es gibt immer wieder Versuche, die Gesellschaft als „kulturell integriert“ zu beschreiben. Wie man mithilfe eines Kulturbezugs Unterschiede zwischen nationalen Gesellschaften markieren kann, zeigt eine Facebook-Debatte zu einem Post von Ruprecht Polenz: „Hierzulande wird über Tempolimit ähnlich emotional diskutiert, wie in den USA über GunControl \#kulturelleIdentität“.

Stefan Nacke: nacke03@web.de 
Kultur gibt immer wieder Anlass für gesellschaftspolitischen Streit, so z. B. in der Debatte um Integration und Leitkultur. Während die eine Seite für Multikulti ist, hat die andere Angst vor kultureller Überfremdung. Kultur wird habituell benutzt als Medium sozialer Distinktion (Hochkultur, Bildungsbürgertum, Arbeiterkultur). Wenn ich mich an meine Studienzeit zurückerinnere, denke ich an die unterschiedlichen Kleidungsstile und Moden von Kommilitonen verschiedener Fachbereiche wie z.B. Theologie und oder Betriebswirtschaft.

Wir sprechen in kultureller Hinsicht über Individualisierung und die Erosion traditioneller Milieus, wie z. B. dem Arbeitermilieu oder dem katholischen Milieu. Wenn wir an das berühmte MoMA (Museum of Modern Art) denken und die an Litfasssäulen groß beworbenen Kunstausstellungen der Museen in den Metropolen, oder wenn in den Zeitungen oder in der Tagesschau über exorbitante Versteigerungserlöse bei Sotheby's berichtet wird, interessieren wir uns für die großen Meister, für Kunstwerke und den ideellen und pekuniären Wert, den sie für Liebhaber haben oder den sie auf dem Markt erzielen. Ganz zu schweigen von den Werbeeinlagen dieser Zeitungen, in denen die neuesten oder die klassischen Designprodukte präsentiert werden, die unsere Wohnkultur bereichern und von den Konzerten, die die Fußballstadien füllen. Evident ist, dass Kultur in aller Munde ist. Was aber hat Kultur mit Bildung zu tun und vor allem mit Weiterbildung und lebenslangem Lernen?

Im Folgenden möchte ich diese Frage in drei Schritten erörtern, indem ich erstens den Kulturbegriff selbst ins Auge fasse und frage, was mit Kultur im engen oder weiteren Sinne gemeint sein kann. Dann greife ich einen aktuellen kultursoziologischen Beitrag auf, der aus gesellschaftstheoretischer und sozialstruktureller Perspektive einen Zusammenhang von Bildung und Kultur postuliert. Und schließlich geht es, daran anschließend, um die notwendigen politischen Konsequenzen.

\section{Der Kulturbegriff}

Wie man am vielfältigen Sprachgebrauch von Alltagskultur über Fußballfankultur bis zur Kulturlandschaft und Kulturtechniken und so weiter sehen kann, hat das Wort „Kultur“ Konjunktur und ist ein vielgebrauchter und deswegen vielleicht ein schillernder Begriff, dessen Bedeutung zu verschwimmen droht. Im Zusammenhang einer Selbstbeschreibung des Kunstsystems ist „Kultur“ für den Soziologen Niklas Luhmann sogar der „schlimmste Begriff, der je gebildet“ worden sei. ${ }^{1}$ Die Spannweite, die er ausfüllen soll, sei viel zu groß. Für die

1 Vgl. Luhmann, Niklas: Die Kunst der Gesellschaft. Frankfurt/M 1997, S. 398. 
Beschreibung des Kunstsystems, das sich selbst gerne mit der Hochkultur identifiziert, eigne er sich nicht, wohl aber als ein Themenvorrat und Semantik- und Bedeutungsreservoir für die gesellschaftliche Selbstbeschreibung: „Kultur ist, so können wir festhalten, das Gedächtnis sozialer Systeme, vor allem des Gesellschaftssystems. Kultur ist, anders gesagt, die Sinnform der Rekursivität sozialer Kommunikation.“2 Ein Bewusstsein von „Kultur“ entsteht, wenn man Luhmann folgt, aus einem Vergleichszusammenhang: zeitlich als historischer Rückblick, sozial als Aufeinandertreffen von Bedeutungen kommunizierenden Populationen und sachlich in den verschiedenen thematischen Funktionsbezügen. Kultur kann als zweite Beschreibung genutzt werden, als weiterer Gesichtspunkt. Dabei überziehe sie alles, was kommuniziert werden kann, mit Kontingenz, sie befreie von Notwendigkeit und verweise auf Alternativen. „Ansprüche auf Authentizität ziehen sich ins Inkommunikable zurück oder werden als Marotte bestimmter Personen oder Gruppen, Ethnien oder Sekten behandelt. “3 Es könne nicht gelingen, einen Sachverhalt „Kultur“ auf der Gegenstandsebene zu fixieren und von anderen Gegenständen zu unterscheiden. Vielmehr konzipiert Luhmann „Kultur“ als eine Perspektive für die Beobachtung von Beobachtern, gleichsam als eine Vogelperspektive auf die Gesellschaft in Form einer Beobachtung zweiter Ordnung.

Soweit ein analytischer Einstieg zum Kulturbegriff aus gesellschaftstheoretischer Sicht. Wie aber gehen Praktiker mit dem Kulturbegriff um? Welche Reichweite soll in Sachen „Kultur“ handlungsleitend sein?

Der für die Beschreibung von Kulturpolitik in Deutschland immer noch einschlägige Abschlussbericht der Enquetekommission „Kultur in Deutschland“ macht sich den weiten Kulturbegriff der UNESCO-Kulturkonferenz von $1982 \mathrm{zu}$ eigen. Er sieht „Kultur“ als die Gesamtheit der unverwechselbaren geistigen, materiellen, intellektuellen und emotionalen Eigenschaften, die eine Gesellschaft oder soziale Gruppe kennzeichnen und die über Kunst und Literatur hinaus auch Lebensformen, Formen des Zusammenlebens, Wertssysteme, Traditionen und Überzeugungen umfasse. ${ }^{4}$ Vor dem Hintergrund, dass Künste sich als Ausdrucksformen erwiesen, in denen Emotionen und konkrete Situationen zur Sprache gebracht werden könnten, sei es wichtig, den Bürgern Gelegenheit

2 Vgl. Ders.: Kultur als historischer Begriff. In: ders.: Gesellschaftsstruktur und Semantik Bd. 4. Frankfurt/M. 1995, S. 47.

3 Ebd. S. 51. Vgl. darüber hinaus zu unterschiedlichen Konzeptionalisierungen von Kultur überblicksweise Levod, Tom: System und Kultur. Warum sich Systemiker mit Kultur beschäftigen sollten. In: Kontext 44,1 (2013), S. 6-21.

4 Vgl. Deutscher Bundestag (Hg.): Kultur in Deutschland. Schlussbericht der Enquete-Kommission des Deutschen Bundestages. Regensburg 2008. 
$\mathrm{zu}$ geben, ihren kulturellen Interessen folgen $\mathrm{zu}$ können, ihre Fähigkeiten zu entwickeln und am kulturellen Leben teilzunehmen. Deswegen sei kulturelle Bildung ein zentrales politisches Aufgabenfeld. Dies nicht zuletzt, weil der aktuelle gesellschaftliche Wandel von der Industrie- zur Wissensgesellschaft sich für manche mit mehr oder weniger gut begründeten Ängsten verbinde. Wegen solcher Exklusionsbefürchtungen, abgehängt zu werden, bedürfe es einer Kulturpolitik, die Prozesse kultureller Partizipation vorantreibe. Diesem breiten Kulturbegriff folgend, entwickelt der Bericht „Kulturpolitik“ als übergreifende Gesellschaftspolitik. Über konkrete Fragen der Kulturpolitik hinaus, geht es um die Gesellschaft als ganze und um die Bedingungen der Möglichkeit, diese Gesellschaft mitzugestalten. Während auf Bundesebene ordnungspolitisch die rechtlichen Rahmenbedingungen, wie Urheberrecht, Sozialversicherungsrecht, etc. für Kunst und Kultur geschaffen würden, zeige sich der besondere Kulturauftrag der Länder und ihre kulturpolitische Zuständigkeit in der Bereitstellung finanzieller Mittel der konkreten Kulturförderung.

\section{Ein aktueller kultursoziologischer Beitrag}

Ein sehr weiter Kulturbegriff wird auch in dem spektakulären Titel „Gesellschaft der Singularitäten“ von Andreas Reckwitz entwickelt, mit dem ich mich im Folgenden auseinandersetzen möchte. Mit seinem erfolgreichen Buch hat Reckwitz bewiesen, dass man auch mit anspruchsvoller Theorie innerhalb eines Jahres sechs Auflagen erzielen kann! Hierfür entfaltet er die fundamentale These, dass die Moderne nur unzureichend als Rationalisierung beschrieben sei. Vielmehr gehe es um eine Gleichzeitigkeit von Rationalisierung und Kulturalisierung. Unter Kulturalisierung versteht er einen Prozess der Bewertung und der Entwertung von Dingen, Zuständen und Phänomenen. Für diesen Vorgang prägt er den Begriff der Valorisierung.

Für den Kultursoziologen hat sich durch die Bildungsexpansion der letzten vier Jahrzehnte und die damit einhergehende Akademisierung breiter gesellschaftlicher Gruppen eine neue gesellschaftliche Mittelschicht entwickelt. Kennzeichen ist ihre Hochschulbildung und ihr hohes kulturelles Kapital: „Authentizität, Selbstverwirklichung, kulturelle Offenheit und Diversität, Lebensqualität und Kreativität sind die Parameter dieses Lebensstils, der auch über die Grenzen ihrer primären Trägergruppe hinaus Strahlkraft erlangt und zu einer Hegemonie geworden ist. Das Milieu der Akademiker, der Hochschulabsolventen und Hochqualifizierten bildet seit den 1980ern Jahren dabei immer weniger eine kleine Elite, sondern macht in den westlichen Gesellschaften - mit wach- 
sender Tendenz - etwa ein Drittel der Bevölkerung aus: Sie ist die neue Mittelklasse. “5

Im Unterschied zur industriell geprägten „organisierten Moderne“ sei in der Spätmoderne ein neuer, ästhetischer Lebensstil entstanden, der sich vor allem am Selbstverwirklichungsparadigma orientiere. Die Angehörigen dieser neuen Mittelschicht führen in der Sorge um Authentizität ein „kuratiertes Leben“. Dabei erfinde sich der Kurator nicht von Grund auf neu, sondern stelle vielmehr zusammen, er mache Dinge zu Ausstellungsstücken, transponiere sein Leben als Kunstwerk. Das Kreative dieser Lebensführung bestehe in der Umgestaltung des Gegebenen, das Neue dieses kreativen Lebensstils meine somit nicht das nie Dagewesene, sondern das relativ Neue: „Das Kuratieren als Querschnittspraxis löst die Querschnittspraxis des Konsums in sich auf. “6 Leben wird in der neuen Mittelschicht zum Medium der Selbstgestaltung und drängt in Zeiten der Digitalisierung und von Social Media nach Darstellung, denn Unsichtbarkeit bedeute den sozialen Tod. Aus der Leistungskultur der industriellen Moderne sei eine Kultur des Erfolgs geworden: es geht um erfolgreiche Selbstverwirklichung. So wird die Lebensführung als Kultur zelebriert, mit dem Ziel, die größtmögliche Fülle des Lebens zu realisieren. Das Selbstverwirklichungssubjekt versucht, möglichst viele Bestandteile seines Alltags einer Kulturalisierung zu unterziehen, wie z. B. Freizeitgestaltung, Wohnungsausstattung, aber auch Arbeit. Arbeit stiftet Identität. Das Selbstverwirklichungssubjekt der neuen Mittelschicht will sie mit intrinsischer Motivation betreiben. Arbeit soll zu sozialer Anerkennung führen und letztlich die Ressourcen für einen kosmopolitischen Lebensstil sichern. Als individuell kuratiertes Lebensgesamtkunstwerk folgt es einer Logik der Besonderung. Diese Orientierung an der Einzigartigkeit wird als Singularisierungsprozess gesamtgesellschaftlich dominant und verändert die Gesellschaft hin zu einer „Gesellschaft der Singularitäten“. Das Allgemeine, die Dinge und gesellschaftlichen Funktionen werden einer weiteren Bewertung unterzogen, werden für die Authentizität des eigenen Lebens als wichtig oder unwichtig be- und entwertet und in Anspruch genommen. Dieser Prozess der Valorisierung beschreibt einen alles umfangenden Prozess der Kulturalisierung. Ausgehend vom Bildungsstatus der neuen Mittelschicht betreibe die Gesellschaft der Singularitäten eine tiefgreifende Kulturalisierung des Sozialen. Während die mit der industriellen bzw. organisierten Moderne verbundene Rationalisierung auf das „Wie“ antworte und auf Funktionalitäten und Effizienzen abstelle, antworte Kulturalisierung auf das „Warum“, auf die Sinnfragen und Motivationen der anspruchsvoll gewordenen

5 Reckwitz, Andreas: Die Gesellschaft der Singularitäten. 6. Aufl. Berlin 2018, S. 275.

6 Ebd., S. 296. 
Menschen. „Das Sinn- und Motivationsproblem, auf das die Kulturalisierung antwortet, ist generell so präsent wie das Effizienz- und Ordnungsproblem - und sobald die Effizienz- und Ordnungsprobleme weniger dringlich geworden sind, tritt es sogar in den Vordergrund.“7

Die von Reckwitz postulierte Spätmoderne vollzieht somit einen Wertewandel und löst tiefgreifende Orientierungsprobleme insbesondere bei der alten, in der industriellen Moderne primär von Facharbeitern gebildeten Mittelschicht aus. Es beginnt ein kulturell induzierter Verdrängungsprozess, dem die alte Mittelschicht zum Opfer falle. Zugleich entsteht mit dem Bildungsprekariat eine neue Unterschicht. Für die nivellierte Mittelstandsgesellschaft der industriellen Moderne stand die Arbeit als Lebensstandardsicherung im Vordergrund. Kennzeichnend für sie war eine vergleichsweise geringe Einkommensungleichheit sowie ein Massenwohlstand. Es gab eine gewisse mit dem Aufstiegsversprechen des Sozialstaats verbundene Bildungsmobilität, und die Wohnviertel waren verhältnismäßig gemischt. Während in der nivellierten Mittelstandsgesellschaft einfache Bildungsabschlüsse Chancen eröffneten und als normaler Durchschnitt galten, werden diese in der Spätmoderne entwertet und ihre Träger zu Niedrigqualifizierten mit extrem begrenzten Möglichkeiten. Der Lebensqualität, die der neuen Mittelschicht das soziale Prestige verschafft, stehen geringe Kulturalisierungschancen des Lebens der unteren, prekären Klasse gegenüber. So kommt es, dass abgewerteten „Provinziellen“ neue Kosmopoliten gegenüberstehen. Die Bildungsexpansion der vergangenen Jahrzehnte hat nicht zu mehr Gleichheit, sondern zu einer neuen, nunmehr kulturellen Spaltung und Segregation der Gesellschaft geführt.

Abgesehen von einer zahlenmäßig winzigen und $\mathrm{zu}$ vernachlässigen Oberschicht ist die Spätmoderne nach Reckwitz eine Drei-Drittel-Gesellschaft geworden, eine Klassengesellschaft mit neuer und alter Mittelschicht und einer prekären neuen Unterschicht. Die alte Mittelschicht, bestehend aus Facharbeitern, gerät in materieller, insbesondere aber in kultureller Hinsicht zunehmend in die Defensive und kultiviert gleichermaßen Abstiegsängste in Richtung neuer Unterschicht. „Die Polarisierung auf der Ebene von Bildung und kulturellem Kapital ist das zentrale Merkmal, welches die Sozialstruktur der spätmodernen Gesellschaft prägt." ${ }^{8}$

Im Wertverlust ehemals angesehener mittlerer Bildungsabschlüsse ist ein Prozess kultureller Entwertung erkennbar. Wenn man materiell auch weiterhin gut situiert sein mag, findet man sich kulturell doch in der Defensive. Während

7 Ebd., S. 87.

8 Ebd., S. 280. 
die alte nivellierte Mittelstandsgesellschaft ihre Verheißungen eines kommoden Lebensstils mittlerer Ansprüche noch für die meisten wahrmachen konnte, verspricht der singularistische Lebensstil der Spätmoderne zwar für die gesamte Gesellschaft Vorbildlichkeit und Attraktivität, aber dieses Versprechen könne eben nicht für alle eingelöst werden. War die alte Industriearbeiterschaft noch stolz auf ihre harte Arbeit und darauf, ihren Lebensunterhalt in Würde zu bestreiten, steht die Lebensform der neuen, ungebildeten Unterklasse gewissermaßen am Rande der Überforderung. Ihre Beziehung zur Arbeit ist rein instrumentell. Man muss sich halt durchbeißen, um sich am Leben zu erhalten. Man muss sich mit langweiligen, formalisierten und standardisierten Jobs abrackern, um ein anständiges Leben führen zu können. Bereits das einfache Herstellen von Normalität ist harte Arbeit. Dass sich die neue Unterklasse als Gruppe der Abgehängten wahrnimmt, die den öffentlich sichtbaren Lebensstil der neuen Mittelklasse nicht erreichen kann, provoziert Gegenreaktionen und Abwehrstrategien, wie man aktuell an den Protesten der sogenannten „Gelbwesten“ in Frankreich erleben kann. Hier liegt der Ansatz eines Distinktionskampfes „unten gegen oben“ oder Establishment gegen das Volk.

\section{Notwendige politische Konsequenzen}

Der soziale Friede unserer Gegenwartsgesellschaft wird durch neue Spaltungen strapaziert, die sich entlang der dominanten gesellschaftlichen Dynamik als neu formierte Kulturkämpfe darstellen. Es geht um die Möglichkeiten, ein wertvolles und gesellschaftlich anerkanntes Leben führen und das Freiheitsversprechen des Bildungsaufstiegs für sich realisieren zu können. Neben der klassischen Kulturpolitik findet eine Kulturalisierung der Politik als ganze statt. Die politische Polarisierung besteht zwischen einer liberalen Perspektive, die kulturelle Öffnung und Vielfalt, Grenzüberschreitung und Kosmopolitismus vertritt und durch die neue Mittelschicht verkörpert wird und einem antiliberalen Kulturkommunitarismus und Kulturessentialismus, der auf Ethnizität, Nationalität, religiösen Fundamentalismus und Rechtspopulismus setzt. Das Ideal der Populisten sind geschlossene Kulturkreise. Der Populismus „beansprucht, den Volkswillen unmittelbar in politische Praxis umzusetzen; er setzt die Möglichkeit und Wünschbarkeit einer Identität zwischen Regierenden und Regierten voraus und arbeitet an einem demokratietheoretischen Modell, das nicht mehr von Pluralität und Repräsentation ausgeht. Vielmehr gibt es aus dieser Perspektive ein kollektives Interesse des Volkes, das ohne Vermittlungsinstanzen unmittelbar in politisches Handeln umzusetzen ist (...) Die Populisten sehen sich in 
diesem Sinne nicht als Repräsentanten, sondern als Teil des Volkes als Spitze einer ,Bewegung' - ,Wir sind das Volk'“. Reckwitz springender Punkt ist, „dass im populistischen Modell der Politik das Volk als Basis der Demokratie homogen gedacht wird (...) Ein Modell sozialer Homogenität suggeriert eine soziale Einheitlichkeit der Lebenssituation der ,eigentlichen’ Bevölkerung (...) Ein Modell kultureller Homogenität suggeriert eine Einheitlichkeit der Werte und Praktiken, eine Nationalkultur (...) Der Rechtspopulismus greift damit maßgeblich auf den Kulturessentialismus zurück“. 9

Angesichts einer solcherart ausgreifenden Logik des Besonderen geht es innerhalb der Gesellschaft der Singularitäten um eine Rekonstruktion des Allgemeinen. Allen voran geht es um die Wiederherstellung einer allgemeinen, d.h. allen gemeinsamen Öffentlichkeit als gemeinsamer Diskurs- und Reflexionsraum. Dafür müssen die durch Filterblasen induzierten Fragmentierungen überwunden werden. ${ }^{10}$ Kulturpolitik ist wahrlich als Gesellschaftspolitik zu entwerfen, insbesondere unter Einbeziehung der Bildungs- und Weiterbildungspolitik. Im föderalen Aufbau der Bundesrepublik sind hier die Länder besonders gefordert. Wie Reckwitz herausgearbeitet hat, unterlaufen die Ökonomie der Singularitäten und die etablierte Kultur der Attraktivität regelmäßig die herkömmlichen Gerechtigkeitsmaßstäbe. Die Gesellschaft der Singularitäten gibt Anlass zur Empörung. Wir dürfen Kulturpolitik nicht naiv auf dem Niveau einer Beobachtung erster Ordnung fortsetzen, der Kulturalisierung der Politik entspricht ein weiter Kulturbegriff. Es geht um neue Regulierungen, mit denen abgewendet werden kann, dass die errungenen Freiheiten nicht aufs Spiel gesetzt werden. Es geht, mit Reckwitz gesprochen, um beides: um das Soziale mit Blick auf Fragen sozialer Ungleichheit sowie des Arbeitsmarktes und um das Kulturelle mit Blick auf die Sicherung allgemeiner kultureller Güter und Normen: „Die Arbeit an der Allgemeinheit des Sozialen und die Arbeit an der Allgemeinheit der Kultur erweisen sich so als zwei Seiten eines politischen doing universality. In beiden Hinsichten könnte im Rahmen des regulativen Liberalismus im Übrigen Instanzen wieder an Bedeutung gewinnen, die im apertistisch-differenziellen Liberalismus minimiert worden sind: die Institutionen des Staates. "11 Damit ist neben den Schulen und Universitäten als Bildungseinrichtungen und den klassischen Kultureinrichtungen wie öffentliche Bibliotheken, Museen, Theater und Konzertsäle insbesondere die gemeinwohlorientierte Weiterbildung angesprochen. Seit 100 Jahren gibt es

9 Ebd., S. 415.

10 Mit dieser Frage beschäftigt sich auch die im Dezember 2018 konstituierte Enquete-Kommission „Subsidiarität und Partizipation. Zur Stärkung der (parlamentarischen) Demokratie im föderalen System aus nordrhein-westfälischer Perspektive“ des Landtags von Nordrhein-Westfalen. 11 Reckwitz 2018, S. 442. 
in Deutschland Volkshochschulen, deren gesellschaftspolitische und damit auch kulturpolitische Relevanz nicht zu unterschätzen ist. ${ }^{12}$ Es geht um eine Stärkung der Erwachsenenbildung, des lebenslangen Lernens, des Nachholens von Schulabschlüssen für neue Chancen am Arbeitsmarkt, es geht um das Erlernen von Sprachen und nicht zuletzt um kulturelle und politische Bildung.

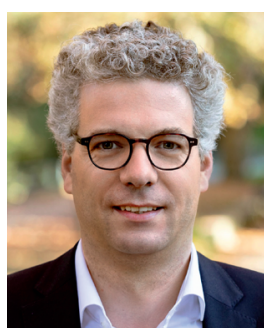

\section{Dr. Stefan Nacke}

Mitglied des Landtags von Nordrhein-Westfalen

Mauritzstr. 4-6

48143 Münster

E-Mail:nacke03@web.de

12 Vgl. z. B. Hinzen, Heribert; Meilhammer, Elisabeth: 100 Jahre Volkshochschule (Bildung und Erziehung, 71 (2018) Heft 2. 